\title{
Effect of Ubumex Combined with AC-T Sequential Chemotherapy on Serum T Cell Subsets, OPN Levels and Quality of Life in Patients with Triple Negative Breast Cancer
}

\author{
Yan Fan, Wenmin Cai, Sawei Li, Lulu Zhang \\ Henan Province Hospital of TCM, Henan, Zhengzhou, 450002
}

Keywords: Triple-Negative Breast Cancer; AC-T Sequential Chemotherapy Regimen; Ubumex; T Lymphocyte Subsets; OPN Levels

\begin{abstract}
Objective: To investigate the application of Ubumex combined with AC-T sequential chemotherapy in the treatment of triple negative breast cancer (TNBC). Methods: From January 2016 to January 2018, 92 patients with TNBC were enrolled. According to the random number table, the patients were divided into observation group and control group, with 46 cases in each group. The control group received a simple AC-T sequential chemotherapy regimen, and the observation group was treated with Ubumex. The clinical efficacy, serum T lymphocyte subsets and osteopontin (OPN) levels and quality of life improvement before and after treatment were compared. Results: The total effective rate of the observation group was $91.30 \%(42 / 46)$ higher than that of the control group 76.09 (35/46, $\mathrm{P}<0.05)$. There was no significant difference in serum $\mathrm{T}$ lymphocyte subsets and OPN levels between the two groups before treatment $(\mathrm{P}>0.05)$. The level of $\mathrm{T}$ lymphocyte subsets in the observation group was higher than that in the control group, and the OPN level was lower than that in the control group $(\mathrm{P}<0.05)$. The improvement rate of quality of life in the observation group was 54.35\% (25/46) higher than that in the control group (32.61\%). Conclusion: Ubumex combined with AC-T sequential chemotherapy in the treatment of TNBC is effective, can effectively improve the body's immune function, down-regulate the expression of serum OPN, and improve the quality of life of patients.
\end{abstract}

\section{Introduction}

Triple negative breast cancer (TNBC) is a special type of breast malignant tumor disease, accounting for about $15 \%$ of all breast cancers. Currently, chemotherapy is the main intervention [1] The AC-T sequential chemotherapy regimen has been widely implemented in the treatment of TNBC. This regimen is given to docetaxel at the same time as anthracycline chemotherapy, which promotes the polymerization of tubulin and inhibits the depolymerization of tubulin. However, some patients with TNBC still have difficulty in achieving ideal therapeutic effects with AC-T sequential chemotherapy. Chemical drugs can impair the immune function of patients and reduce the tolerance to drugs, which leads to a significant decline in the quality of life and cause clinical attention. Ubumeex can enhance immune function, usually used for adjuvant treatment of anticancer chemoradiotherapy. This product not only interferes with tumor cell metabolism, promotes tumor cell apoptosis, but also activates cellular immune function and stimulates cytokine production. This study selected 92 patients with TNBC in our hospital to discuss the clinical value of Ubumex combined with AC-T sequential chemotherapy.

\section{Materials and Methods}

General Information from January 2016 to January 2018, 92 patients with TNBC in our hospital were enrolled in the observation group and the control group according to the random number table method. 46 patients in the observation group, aged 24 to 69 years, mean $(49.58 \pm 9.23)$ years; pathological classification: 23 cases of invasive ductal carcinoma, 9 cases of medullary carcinoma, 14 cases of adenocarcinoma. Forty-six patients in the control group, aged 25-68 years, mean (48.74 \pm 8.97$)$ years old; pathological type: 24 cases of invasive ductal carcinoma, 10 cases of 
medullary carcinoma, 12 cases of adenocarcinoma. There was no significant difference in baseline data between the two groups $(\mathrm{P}>0.05)$. This study meets the relevant requirements of the Helsinki Declaration of the World Medical Association and is approved by the hospital ethics committee.

Inclusion criteria for inclusion criteria: (1) Conform to the diagnostic criteria of TNBC in Surgery [2], immunological examination of progesterone receptor, estrogen receptor, human epidermal growth factor receptor-2 showed negative; (2 Carnival functional status (KPS) score $\geq 70$ points; (3) no cognitive impairment, informed consent. Exclusion criteria: (1) combined with autoimmune diseases; (2) liver, kidney and other important organ dysfunction; (3) combined with blood system diseases; (4) pregnant or lactating women.

Methods (1) The control group was given a simple AC-T sequential chemotherapy regimen, cyclophosphamide (Shanxi Pude Pharmaceutical Co., Ltd., National Pharmaceutical Standard H14023686) 300 mg/m2, epirubicin (Haizheng Pfizer) Pharmaceutical Co., Ltd., Guoji Zhunzi $\mathrm{H} 20030260) 75 \mathrm{mg} / \mathrm{m} 2$, all used in the intravenous infusion on the 1st day of the chemotherapy cycle, 21 days as a chemotherapy cycle, a total of 4 cycles; followed by sequential administration and Dorsey Hesai (Shanghai Shangyao Xinya Pharmaceutical Co., Ltd., Guoji Zhunzi H20080407) $100 \mathrm{mg} / \mathrm{m} 2$, intravenous infusion on the 1st day of the chemotherapy cycle, and 21 days as a chemotherapy cycle, a total of 4 cycles. (2) During the course of AC-T sequential chemotherapy, the observation group was treated with Ubumex (Nanjing Group Chuan Kang Pharmaceutical Co., Ltd., Guoyao Zhunzi H20090293), orally, 30 mg/time, once a day.

Laboratory index examination method Take $6 \mathrm{ml}$ of venous blood in the fasting state of the patient, and aliquot 2 to detect: (1) T lymphocyte subset: detected by EpicsXL flow cytometer (Beckman Coulter, USA) CD3+, CD4+ and CD8+ were calculated for CD4+/CD8+ values, and the kit was provided by Shanghai Baili Biotechnology Co., Ltd. (2) Serum osteopontin (OPN) level: After centrifugation (3000 r/min, $20 \mathrm{~min}$ ), the upper serum was taken for examination and detected by enzyme-linked immunosorbent assay. The instrument was Bio-RAD550 microplate reader (Bio-American Bio- RAD), the kit is provided by Shanghai Yifeng Biotechnology Co., Ltd.

Efficacy evaluation criteria the tumor completely disappeared or the lesion volume decreased $\geq 75 \%$ for complete remission; the lesion volume was reduced by $>50 \%$ for partial remission; the lesion volume was reduced by $25 \%$ to $50 \%$ for stability; the lesion volume was not reduced or even increased. Trends, or new lesions appear as progress; total effective rate $=$ (complete remission + partial remission) / total number of cases $\times 100 \%$ [3].

(1) Clinical efficacy. (2) Level of laboratory indicators. Serum T lymphocyte subsets (CD3+, CD4+, CD4+/CD8+) and OPN levels were compared before and after treatment. (3) Quality of life. According to the change of KPS score before and after treatment, the KPS score was reduced by more than 10 points before treatment, and the quality of life was reduced. It was improved by no less than 10 points before treatment, and it was stable between the two [4].

Statistical analysis Statistical analysis by SPSS18.0 software, measurement data $( \pm \mathrm{s}), \mathrm{t}$ test, count data $\mathrm{n}(\%), \chi^{2}$ test, $\mathrm{P}<0.05$ for the difference was statistically significant.

\section{Results}

\subsection{Clinical Efficacy.}

The total effective rate of the observation group was $91.30 \%$ higher than that of the control group $76.09(\mathrm{P}<0.05)$, as shown in Table 1.

Table 1 Comparison of clinical effects between the two groups [n (\%)]

\begin{tabular}{lllllll}
\hline Group & $\begin{array}{l}\text { Number } \\
\text { of cases }\end{array}$ & $\begin{array}{l}\text { complete } \\
\text { relief }\end{array}$ & Partial relief & stable & progress & $\begin{array}{l}\text { Total } \\
\text { efficiency }\end{array}$ \\
\hline $\begin{array}{l}\text { Observation } \\
\text { group }\end{array}$ & 46 & $29(63.04)$ & $13(28.26)$ & $3(6.52)$ & $1(2.17)$ & $42(91.30)$ \\
$\begin{array}{l}\text { Control group } \\
\chi^{2}\end{array}$ & 46 & $21(45.65)$ & $14(30.43)$ & $7(15.22)$ & $4(8.70)$ & $\begin{array}{l}35(76.09) \\
P\end{array}$ \\
\hline
\end{tabular}




\subsection{Laboratory Indicators.}

There was no significant difference in serum T lymphocyte subsets and OPN levels between the two groups before treatment $(\mathrm{P}>0.05)$. After treatment, the $\mathrm{T}$ lymphocyte subsets in the observation group were higher than the control group, and the OPN level was lower than the control group (P). $<0.05)$, see Table 2 .

Table 2 Comparison of serum T lymphocyte subsets and OPN levels before and after treatment in both groups $( \pm s)$

\begin{tabular}{|c|c|c|c|c|c|c|}
\hline \multirow{2}{*}{ time } & \multirow{2}{*}{ Group } & \multirow{2}{*}{$\begin{array}{l}\text { Number } \\
\text { of cases }\end{array}$} & \multicolumn{3}{|c|}{ TLymphocyte subset } & \multirow{2}{*}{ OPN(pg/ml) } \\
\hline & & & $\mathrm{CD}^{+}(\%)$ & $\mathrm{CD}^{+}(\%)$ & $\mathrm{CD}^{+} / \mathrm{CD}^{+}$ & \\
\hline \multirow{4}{*}{$\begin{array}{l}\text { Before } \\
\text { treatment }\end{array}$} & $\begin{array}{l}\text { Observation } \\
\text { group }\end{array}$ & 46 & $64.19 \pm 2.34$ & $36.07 \pm 1.25$ & $1.13 \pm 0.18$ & $724.54 \pm 25.63$ \\
\hline & $\begin{array}{l}\text { Control } \\
\text { group }\end{array}$ & 46 & $63.25 \pm 2.41$ & $35.79 \pm 1.36$ & $1.15 \pm 0.19$ & $722.69 \pm 27.04$ \\
\hline & $t$ & & 1.898 & 1.028 & 0.518 & 0.337 \\
\hline & $P$ & & 0.061 & 0.307 & 0.606 & 0.737 \\
\hline \multirow{4}{*}{$\begin{array}{l}\text { After } \\
\text { treatment }\end{array}$} & $\begin{array}{l}\text { Observation } \\
\text { group }\end{array}$ & 46 & $71.02 \pm 3.01$ & $40.02 \pm 1.43$ & $1.62 \pm 0.25$ & $586.03 \pm 31.02$ \\
\hline & $\begin{array}{l}\text { Control } \\
\text { group }\end{array}$ & 46 & $59.27 \pm 2.52$ & $32.34 \pm 1.05$ & $1.02 \pm 0.21$ & $652.49 \pm 34.17$ \\
\hline & $t$ & & 20.301 & 29.361 & 12.464 & 9.767 \\
\hline & $P$ & & 0.000 & 0.000 & 0.000 & 0.000 \\
\hline
\end{tabular}

The quality of life improvement rate of observation group was $54.35 \%$ higher than that of the control group (32.61\%, $\mathrm{P}<0.05$ ), see Table 3 .

Table 3 Comparison of quality of life between the two groups [n (\%)]

\begin{tabular}{lllll}
\hline Group & $\begin{array}{l}\text { Number of } \\
\text { cases }\end{array}$ & improve & stable & reduce \\
\hline Observation & 46 & $25(54.35)$ & $16(34.78)$ & $5(10.87)$ \\
group & & $15(32.61)$ & $19(41.30)$ & $12(26.09)$ \\
Control group & 46 & 4.423 & 0.415 & 3.536 \\
$\chi^{2}$ & & 0.036 & 0.519 & 0.060 \\
$P$ & & &
\end{tabular}

\section{Discussion}

Chemotherapy is the main method for the treatment of TNBC at this stage, and the first-line chemotherapy in the clinic advocates the combination of anthracyclines and taxanes to produce tumor cells through different action machines, and achieve higher clinical remission rate. No cross-resistance, play a synergistic role [5]. Based on this, the AC-T sequential chemotherapy regimen has been widely used in the treatment of TNBC, and the effect has been recognized.

However, AC-T sequential chemotherapy can effectively damage the normal cell tissue function while effectively killing the tumor cells, which not only increases the side effects of chemotherapy, but also impairs part of the body's immune function to some extent. It has been reported in the literature that immunodeficiency can promote the development of malignant tumors, and the pathological changes caused by tumors provide a microenvironment for immunodeficiency, destroying the production of anti-tumor immunity, resulting in increased treatment difficulty, affecting the prognosis of patients, and leading to a significant decline in the quality of life [6]. Therefore, during the course of AC-T sequential chemotherapy in TNBC, it is important to choose an adjuvant treatment program that can enhance the immunity of the body, which can ensure the effect of chemotherapy and promote the improvement of prognosis. Ubumex is a competitive reversible protease inhibitor. Pharmacological studies have shown that this product has a significant role in promoting $\mathrm{T}$ lymphocyte function, can effectively improve the natural killing ability of NK 
cells, and fully play the role of immune regulation [7]. In addition, umbrel can also exert competitive and selective inhibition on aminopeptidase B and leucine aminopeptidase activities, thereby preventing tumor cells from multiplying and proliferating, so as to accelerate the apoptosis of cancer cells, so it is also significant. This study combined with Ubumex and AC-T sequential chemotherapy to treat TNBC, the results showed that the total effective rate of the observation group after treatment was $91.30 \%$, and the $\mathrm{T}$ lymphocyte subsets level was better than the control group $(\mathrm{P}<0.05)$. The program can further improve the therapeutic effect of TNBC, reduce the effect of chemotherapy on the immune function of patients, and improve the immunity of the body. The analysis may be related to the anti-tumor effect and immune regulation of umbrel.

In recent years, with the deep research of molecular biology, OPN has been found to be a secretory phosphorylated glycoprotein, which plays an important role in the occurrence and progression of breast cancer, so it can be used as an important indicator for early diagnosis and prognosis of disease [8]. In this study, the serum OPN level in the observation group was lower than that in the control group $(\mathrm{P}<0.05)$, and the feasibility and effectiveness of Ubumemate combined with AC-T sequential chemotherapy regimen in the treatment of TNBC were further confirmed by objective indicators. The results of this study also showed that the improvement rate of quality of life in the observation group after treatment was better than that in the control group $(\mathrm{P}<0.05)$, suggesting that the above combination scheme has positive significance for improving the quality of life of patients. Disease progression and reduction of immune function caused by chemotherapy drugs, side effects, etc. can have a significant impact on the quality of life of patients with TNBC. On the basis of AC-T sequential chemotherapy, supplemented with Ubumex, it can effectively improve the disease control rate. And enhance the body's immune function, so that patients' tolerance to drug side effects is improved, which is beneficial to alleviate the impact of many factors on the quality of life, and thus improve the quality of life.

In summary, Ubumex combined with AC-T sequential chemotherapy regimen has a significant effect on TNBC, which can effectively improve the body's immune function and down-regulate the expression of serum OPN, which is conducive to improving the quality of life of patients.

\section{References}

[1] Yuan Yewei, Sun Zhijun. The latest treatment and research progress of triple negative breast cancer [J].Chongqing Medical Journal, 2015, 44(16):2279-2282.

[2] Chen Xiaoping, Wang Jianping. Surgery [M]. 8th ed. Beijing: People's Medical Publishing House, 2013: 256-260.

[3] Ren Ning, Cheng Weigang, Wei Limin. Clinical study of Ubumex combined with XEC chemotherapy in the treatment of triple-negative breast cancer [J]. Modern Medicine and Clinical, 2018, 33 (1): 128-133.

[4] Hu Dong. The effect of Wuhong Decoction on bone marrow suppression and quality of life after chemotherapy in breast cancer [J]. New Chinese medicine, 2015, 47(10):171-173.

[5] Ni Chen, Li Ting, Wu Zhenhua, et al. Progress in chemotherapy of triple negative breast cancer [J]. Chinese Journal of Cancer, 2014, 24(4): 316-320.

[6] Wang Yu, Cui Shuzhong. Research progress on immune function status and immunotherapy in patients with malignant tumors [J]. Chinese Journal of Clinical Oncology, 2014, 41(13): 876-879.

[7] Dong Xiaojian, Liu Chunliang, Ge Mei, et al. Effects of Ubumexide on immune cell function in mice [J]. Chinese Journal of Clinical Pharmacology, 2014, 30(7): 607-610.

[8] Huang Wen, Meng Gang, Chen Qing. Expression of OPN, CD44v6 and CD10 in breast cancer and its clinical significance [J]. Journal of Clinical and Experimental Pathology, 2016, 32(1): 23-28. 